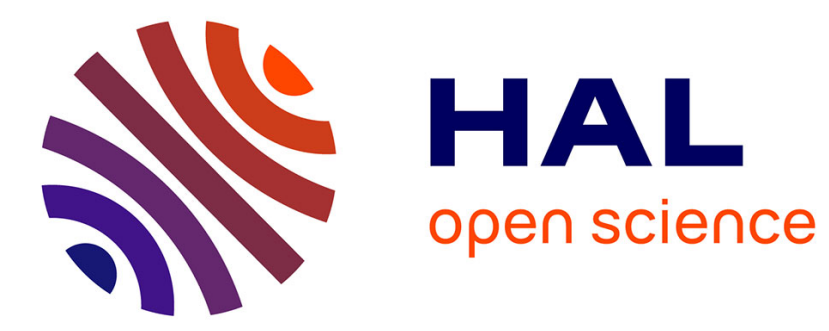

\title{
A Survey on Rapidly Deployable Solutions for Post-disaster Networks
}

Karen Miranda, Antonella Molinaro, Tahiry Razafindralambo

\section{To cite this version:}

Karen Miranda, Antonella Molinaro, Tahiry Razafindralambo. A Survey on Rapidly Deployable Solutions for Post-disaster Networks. IEEE Communications Magazine, 2016, 10.1109/MCOM.2016.7452275 . hal-01285617

\section{HAL Id: hal-01285617 \\ https://hal.inria.fr/hal-01285617}

Submitted on 21 Apr 2016

HAL is a multi-disciplinary open access archive for the deposit and dissemination of scientific research documents, whether they are published or not. The documents may come from teaching and research institutions in France or abroad, or from public or private research centers.
L'archive ouverte pluridisciplinaire HAL, est destinée au dépôt et à la diffusion de documents scientifiques de niveau recherche, publiés ou non, émanant des établissements d'enseignement et de recherche français ou étrangers, des laboratoires publics ou privés. 


\title{
A Survey on Rapidly Deployable Solutions for Post-disaster Networks
}

\author{
Karen Miranda* ${ }^{1}$, Antonella Molinaro ${ }^{2}$, and Tahiry Razafindralambo ${ }^{3}$ \\ ${ }^{1}$ Metropolitan Autonomous University - Cuajimalpa, Mexico \\ ${ }^{2}$ University Mediterranea of Reggio Calabria, Italy \\ ${ }^{3}$ Inria Lille - Nord Europe, France
}

\begin{abstract}
In post-disaster scenarios, e.g., after earthquakes or floods, the traditional communication infrastructure may be unavailable or seriously disrupted and overloaded; therefore, rapidly deployable network solutions are needed to restore connectivity and provide assistance to users and first responders in the incident area. This work surveys the solutions proposed to address the deployment of a network without any a priori knowledge about the communication environment for critical communications. The design of such a network should also allow for quick, flexible, scalable, and resilient deployment with minimal human intervention.
\end{abstract}

\section{INTRODUCTION}

In September 2014, hurricane Odile hit Mexico's Baja California coasts. The category four hurricane devastated several towns, where trees and electricity poles collapsed, causing a general blackout. Following this event, most of the cities hit by the hurricane lost communication services with the outside. This is just one of many possible examples of natural or man-made disasters whose occurrence and consequential damage are difficult to predict. As a result of such a disaster, the communication infrastructure may very often be totally or partially destroyed, transforming the affected zone into an isolated information island. In addition to the infrastructure destruction, the remaining communication networks, e.g., mobile networks, may be insufficient because of the large number of people attempting to call emergency services or communicate with their relatives [1].

After a disaster, providing connectivity among the rescue teams, e.g., firemen, policemen, or paramedics, becomes a crucial task to allow the first responders to report their findings and coordinate their rescue tactics. Therefore, from the networking point of view, the goal is to restore connectivity at least temporarily to provide such communication services. One approach to overcoming such problems is to organize and execute the dissemination of network components, such as routers, access points, or relays, to replace those that were destroyed or to create a network on demand [2].

However, deploying a network under critical conditions presents an important set of challenges. First, the proposed network must be deployed without any a priori knowledge about the environment; therefore, its deployment must be performed on the fly and as rapidly as possible,; i.e., the network must be set up quickly, almost in real time, to replace the damaged portion of the infrastructure or to alleviate traffic congestion. Second, the network must be adaptive, self-reconfigurable, flexible, scalable, and energy efficient to cope with unknown dynamic environments and battery-powered wireless devices; that is, the network must be set up on demand in accordance with the location and current needs. Problems of this type have motivated a long-term research effort on an approach to network component deployment called Rapidly Deployable Networks (RDN). A rapidly deployable network, also known as an impromptu network or spontaneous network, is an adaptive, mobile communications system that can be easily extended [3]. The purpose of an RDN is to temporarily replace the infrastructure damaged after a disaster to guarantee emergency communications among the rescue command center, the first responders and, if possible, the

\footnotetext{
* Part of this work was performed when Karen Miranda was with the FUN project-team at Inria Lille - Nord Europe
} 
victims inside the disaster zone. Restoring permanent communications for the affected population is beyond the scope of this work.

In parallel, considerable effort has been directed toward improving the capabilities of various Professional Mobile Radio (PMR) technologies, such as the ETSI TETRA Enhanced Data Service (TEDS), which still has major limitations with regard to the requirements of new emergency applications. In the U.S., the FCC has identified Long-Term Evolution (LTE) as the technology to be used for public safety networks in the future, and this trend is receiving worldwide consensus. However, key features must still be implemented in LTE before it can satisfy the requirements of public safety systems. Meanwhile, traditional PMRs will be used for mission-critical voice communications, whereas novel technologies will be used for data transfer.

This article summarizes the main requirements for the rapid deployment of network solutions in disaster scenarios and presents a classification of the existing proposals. We describe the main features of such proposals and provide an analysis of the advantages and weaknesses of several approaches.

\section{REQUIREMENTS}

Impromptu networks use wireless technology, which enables flexible, scalable, and mobile deployment with little or no planning in advance. Even if RDNs are a temporary solution, their design must satisfy certain minimal requirements to provide and maintain sustainable communication for first responders.

In general, the most representative requirements for RDN design cited in the literature are as follows:

- Resilience - Resilience is the ability to provide and maintain an acceptable level of service in the face of faults and challenges to normal operation. Hence, the network must provide and maintain essential services under adverse conditions as well as allow for rapid and full service recovery.

- Basic service set - Carrying voice communication is traditionally the main task of a PMR network. However, first responders may need to exchange up-to-date position information, maps, images, and other locally monitored data to coordinate operations. Furthermore, depending on the capabilities of the deployed network, it might be advisable to support video streaming restricted to specific cases for rescue purposes.

- Self-* - Self-capabilities, such as self-organization, self-optimization, and self-healing, help to reduce the need for human intervention in network management, improve process automation, and improve the network's reliability.

- Mobility - Mobile nodes facilitate the deployment and redeployment of the network. Thereby, the network topology may be tailored to the incident zone conditions; moreover, the positions of the nodes can be modified to improve network performance.

- Interoperability - To the greatest extent possible, the deployed network should be able to work compatibly with other heterogeneous undamaged network systems to extend the coverage area and the offered services. This requirement is a very desirable feature of an RDN, although its accomplishment depends on the impromptu conditions under which the network is deployed.

The next section introduces the main wireless technologies that satisfies the requirements just described.

\section{WIRELESS TECHNOLOGIES}

Wireless networks (WNs) have achieved great success because they are, in general, flexible, low cost, easily deployable, and scalable and can dynamically self-organize. These characteristics make wireless networks well suited for military, emergency, disaster, and community applications. In particular, ad hoc networks are an interesting solution for disaster relief scenarios because they are composed of wireless mobile and/or static nodes capable of self-organizing and creating temporary and arbitrary topologies without any pre-existing infrastructure providing interconnections between nodes [4]. At present, by virtue of protocols such as Bluetooth, ZigBee, HiperLAN, and IEEE 802.11x, mobile ad hoc networks (MANET) are easy to assemble. 
Similarly, wireless mesh networks (WMNs) are also a viable solution for rapid network deployment; they are typically based on the IEEE 802.11 s amendment. A WMN is a multi-hop wireless network in which participant nodes connect via redundant interconnections and cooperate with one another to route packets. In a mesh network, a particular set of nodes is dedicated to forwarding the traffic of the other nodes, forming a wireless backhaul that may be considered "infrastructure". As such, WMNs have certain specific peculiarities: $i$ ) multi-hop capability to provide greater coverage and non-line-ofsight (NLOS) communications; ii) self-forming, self-healing, and self-organizing capabilities to provide gradual growth and enhanced performance; and iii) distributed management. WMNs have been used to extend the coverage of public access points and to provide low-cost connectivity to mobile users, a useful property in the context of RDNs. The main advantage of using WMNs for spontaneous networking is the inherent resilience achieved through multi-hop communication, in which nodes are connected by several links and transmit data from source to destination by relaying the necessary packets among themselves. Hence, in the case of failure, it is possible to choose alternative paths by exploiting the self-healing and reconfigurability of the mesh to avoid disconnection [5].

Recently, Cognitive Radio Networks (CRNs) have gained popularity and become promising candidates for exploiting opportunistically available radio interfaces and frequencies in the deployment field. Cognitive radio techniques offer the capability of using or sharing the spectrum in an opportunistic manner, thus allowing them to operate on the best available channel. More specifically, cognitive radio technology enables (1) spectrum sensing (i.e., the determination of which portions of the spectrum are available and the detection of the presence of primary users when a user is operating in a licensed band) and (2) spectrum management (i.e., the selection of the best available channel). Because CRNs have adjustable transmission frequencies, they offer interesting features that are useful in post-disaster scenarios, including self-organization, heterogeneity management, adaptability, reconfigurability, and robustness [6].

In the literature, several proposals have been presented for the rapid deployment of a network as a response to emergency circumstances. These solutions aim to restore connectivity where the existing network infrastructure has been destroyed or never existed. These proposals are mostly based on WMN, ad hoc, and cellular technologies, and they adopt various approaches to determining how, where, and when the devices composing the RDN must be deployed.

\section{RAPIDLy DePloyable NeTWORKS}

Evans et al. introduced the concept of RDNs in 1998 to maintain connectivity among military troops constantly moving through unknown territories [3]. The fundamental idea was to deploy a network infrastructure in an impromptu manner to provide communication services for military applications based on asynchronous transfer mode (ATM) technology.

After this pioneering work, several deployment schemes were investigated, focusing primarily on emergency communications to connect first responders to incident command centers and, if possible, to victims. Hence, we identify two major categories of such schemes: a) metropolitan area approaches and b) local area approaches. The former aims to cover a wide area, and in most cases, the RDN is part of a larger emergency system. Meanwhile, the latter aims to tailor the network topology to each particular scenario and to any eventual changes in the environment. We will discuss each category separately.

\section{Metropolitan AREA APPROACH}

The metropolitan area approach is oriented toward deploying network nodes and devices to cover a large incident area. This approach adopts a layered composition in which the first responders are connected to base stations (BSs) or access points (APs) that are mostly carried by vehicles, such as ambulances, police cars, or fire trucks. Each of these BSs is connected to an aerial or aerospace node, for example, an airplane or a satellite, which, in turn, connects the incident zone to the command center [7]. This architecture is depicted in Figure 1, which illustrates police cars provided with APs such that each AP covers a given area for first-responder communication. 
Because the assets of the rescue forces are used directly, solutions of the type just described are often implemented in broader systems that may include $i$ ) a monitoring tool for zone surveillance before, during, and after a disaster; ii) a public alarm system to warn the population of possible natural disasters; iii) communication infrastructure for disaster recovery scenarios, such as RDNs; iv) communication infrastructure for information dissemination and exchange between the disaster zone and elsewhere; and v) a system for processing and analyzing information about the disaster. Several examples of such systems are the Communications for enHanced envirOnmental RISk management and citizens safeTy (CHORIST) system [8], the Wireless Deployable Network System (WIDENS) project [9], and the Virtual Cell Layout (VCL) scheme [10]; of these, the latter was proposed for military communications. Such systems are based on cluster-based organization in a layered architecture, in which there is a cluster head for each group of first responders. Every cluster head acts as a bridge between the first responders and the access points carried by the vehicles (see Figure 1). Likewise, at the AP level, there is at least one cluster head connecting the remainder of the nodes to an upper layer, such as a satellite acting as a gateway.

CHORIST proposes to form a mesh network using wireless routers carried by emergency vehicles [8]. The vehicles are automatically connected in a peer-to-peer fashion and form a self-configuring intervehicular core. At the edges, the mobile radios carried by the first responders are connected to the closest router via Wi-Fi and create local cells. The remote connection to the command center is established through an IP backbone.

Similarly, WIDENS proposes a cross-layer approach coupled with a cluster-based architecture to provide high-bit-rate service using ad hoc hot spots. This approach includes PHY, MAC, and network levels. The physical layer relies on reconfigurable Orthogonal Frequency-Division Multiple Access (OFDMA) with multi-antenna capability (Multiple Input, Multiple Output; MIMO) to provide data transport services. In addition, this approach includes a MAC layer organized in clusters, where in a given set of nodes, the cluster head is the best located node. Relay nodes are used to ensure interconnectivity between clusters, and gateway nodes are used to connect the created network to other networks. The cornerstone of this proposal is the terminode concept, which means that each node in the network is able to perform the functions of a cluster head, relay, router, or gateway depending on its location and service requirements. The terminodes may dynamically change their roles to take advantage of the cross-layer approach and improve network performance.

Finally, the Virtual Cell Layout scheme for tactical communications divides a given coverage area into fixed virtual cells, similarly to cellular networks $(3 \mathrm{G})$. The access points use VCL resources to create an overlaid real cell that moves over the virtual cells. Manpack Radios (MPRs) form small cells, and each cell has a node that plays the role of an MPR cluster head. These MPR cells are grouped into a larger cell through Radio Access Points (RAPs). Finally, all cells are connected through a satellite or an Unmanned Aerial Vehicle (UAV). The VCL approach focuses on adapting 3G technologies to tactical systems. Accordingly, the authors propose procedures for managing overlaid cells, handoff, and MPR organization.

Recently, the SALICE (Satellite-Assisted LocalIzation and Communication system for Emergency services) Project [11] has been focusing on an integrated navigation (NAV)/communication (COM) reconfigurable system architecture for emergency scenarios. In this architecture, rescuers within the incident area network are organized in teams or clusters. Localization techniques, software-defined radio (SDR) and cognitive radio NAV/COM devices, the integration of satellites and High-Altitude Platforms (HAPs) into rescue services, and the adoption of heterogeneous solutions in the intervention area are the main research topics of the project.

In summary, the metropolitan area approach provides broadband connectivity to rescue teams in largescale scenarios. Nevertheless, this approach offers limited opportunities for redeployment or improvement on demand because the vehicles carrying the nodes have constrained mobility; therefore, their locations may not be optimal for covering all mobile first responders operating inside the incident zone. 


\section{LOCAL AREA APPROACH}

In contrast to the metropolitan area approach, the local area approach aims to provide services in situ; i.e., its intent is to create a dedicated network in a target zone through distributed, inexpensive, on-demand adaptive, and on-the-fly solutions. Hence, the deployment algorithms must be capable of operating without any previous information about the environment. Although all of the various approaches described thus far in this article must consider the so-called SWaP (Size, Weight, and Power) requirements, the functionality of local area approaches depends on these factors particularly strongly. Thus, low SWaP features are highly desirable when implementing an RDN. We introduce two types of mechanisms classified based on their deployment strategies: breadcrumb-based and mobile-backbone-based approaches, which are described in the following sections.

\section{A. Breadcrumb-based approaches}

The term "breadcrumbs" is a reference to the well-known fairy tale "Hansel and Gretel", in which Hansel uses breadcrumbs to trace the way back home. In the context of RDNs, breadcrumbs are small and inexpensive devices that act as relays; i.e., their only goal is to forward packets between edge nodes. Thus, in a given emergency, first responders are provided with several breadcrumb devices and a mobile radio. Following Hansel's example, the mobile users must drop these devices at regular intervals while exploring the emergency zone to maintain connectivity with the command center. The relays are dropped on demand to create a static ad hoc backbone adapted to the environmental dynamics. Figure 2 depicts an example of a breadcrumb-based network; the command center maintains communication with the mobile users through relays dropped by the first responders, thus enlarging the coverage area.

Among the advantages offered by the breadcrumb-based approach, we find that it 1) enables the on-demand creation of a multi-hop network, 2) allows for communication among the first responders as soon as the relays are deployed, 3) allows for communication with isolated relays, 4) guarantees reliable communications, 5) offers an increased coverage area, and 6) reduces the probability of network partitioning. Here, 'reliability,' as cited in (4), refers to the probability that a message transmitted on a link will be successfully received, just as this term is defined in [12]. Because the deployment decisions are made in real time without any knowledge about the final network topology, the main concern to be addressed is when and/or where to place the relays to establish and maintain connectivity for the first responders. A naïve approach is to establish simple, static rules for dropping relays, for example, at any given distance, one relay per floor, or every three doors. However, such rules guarantee only that the relays will lie within each other's communication ranges and do not consider physical phenomena such as interference, channel fading, background noise, or the hidden terminal problem [12], [13]. Thus, the goal is to develop a deployment decision process that maximizes network performance.

Extensive research has been conducted to address the deployment decision problem. The various proposals each describe their own deployment algorithms; nevertheless, they share several common characteristics. The algorithms monitor the link quality through measurements of the received signal strength indicator (RSSI) [14], signal-to-noise ratio (SNR) [12], or bandwidth [13]. Such measurements may be obtained by means of probe packets artificially injected into the network or based on control messages obtained directly from the routing protocol. A threshold for triggering a deployment event is set. Once the link quality drops below this threshold, the user must drop a new relay. For instance, the algorithms presented in [12] and [14] use a pre-defined threshold for all applications, whereas that in [13] sets the threshold based on the bandwidth required for each application. Recall that deployment is performed manually by the first responder; thus, to notify the user, a warning signal such as a light or a sound is also implemented. Therefore, an efficient deployment algorithm must be capable of monitoring changes in the network to satisfy the relays' needs.

Bao and Lee proposed a collaborative algorithm that introduces two types of control information, one to request and one to announce the deployment of a relay [14]. Such control messages are added to the control packet header from the routing protocol. Thus, relay deployment is triggered either because the 
mobile user detects link quality degradation or because it receives an explicit deployment request from its neighbors. The latter case occurs when a mobile user runs out of relays; hence, the mobile user sends a request message to its neighbors for another user to drop a relay. Once the deployment decision is made, the candidates to be deployed broadcast announcement messages.

Similarly, Souryal et al. proposed an algorithm based on a rapid evaluation of the physical layer performed by the mobile relay [12]. The relay constantly broadcasts probe packets to the previously dropped relays. When a relay is within communication range, it responds with an acknowledgment packet. Then, the relay measures the SNR through ACK reception; if the maximum ACK value falls below the threshold level, a new relay must be dropped.

The breadcrumb approach is well suited to extending communication coverage for first responders in indoor scenarios. Moreover, the breadcrumb backbone may be easily deployed by following the dropping rules. Therefore, it is possible to adapt the network topology to the sizes and configurations of different scenarios as well as to create a resilient network by adding a few redundant relays. However, this approach does not offer any possibility of redeployment because the relays have no mobility of their own. Indeed, in the proposals mentioned above, the deployment action depends on the users; i.e., the users must take direct action by dropping the devices. However, this is not necessarily the ideal case. When the first responders enter an emergency zone, their first priority is not relay deployment, and therefore, they may bypass dropping a relay or may simply miss the deployment signal. To overcome this problem, an automatic breadcrumb dispenser is proposed in [15]. Along with the dispenser, an algorithm based on a utility function is proposed. This algorithm attempts to optimize the trade-off between the improvement to the communication link accomplished by deploying a new relay and the number of remaining relays.

Finally, given that the nodes are static, low SWaP values may be achieved at the expense of sacrificing mobility and weight. Thus, the design of such an impromptu deployed network should take this trade-off into account.

\section{B. Mobile robotic backbone approach}

The breadcrumb approach has achieved great success by virtue of its flexibility and cost efficiency; however, the relays remain static once deployed, which is the primary disadvantage of this strategy. That is, if the environmental conditions eventually change, the breadcrumb network may not be able to adapt to the new conditions. In recent years, a mechanism similar to the breadcrumb approach has been developed. In this approach, the relays are provided with autonomous mobility for self-deployment. This capability allows the mobile relays to adjust their own locations on demand. This mobile robotic approach was enabled by advances in robotics and automation.

Similar to the breadcrumb approach, the purpose of a robotic backbone network is to provide communication connectivity to mobile users with minimal human intervention during deployment. The fleet of mobile relays must be able to organize and deploy on their own and, if possible, to optimize network performance. Hereafter, we use the terms 'robotic backbone network' and 'robot-based wireless relay network' interchangeably. In Figure 3, we illustrate the concept of a mobile-robot-based network. In brief, the mobile relay devices are placed in an initial configuration, either clustered together or not. By following a deployment algorithm, they will self-spread across the target zone, creating a wireless backbone.

We identify two types of schemes in this category. Schemes of the first type are based on strategies related to chain formation, i.e., the robots follow each other along a chain (see Figure 3). Among these, Pezeshkian et al. proposed an initial convoy arrangement in which the relays follow a robot leader one after the other, forming a line [16]. When the degradation in the RSSI reaches a certain threshold, the farthest relay in the line will stop and convert into a static relay. This process is repeated until all relays have become static nodes.

Similarly, Nguyen et al. investigated the case of two APs outside each other's transmission range in a wireless mesh network [17]. A chain of relays is deployed to restore the connectivity between such APs. 
The authors' proposed algorithm considers three types of relays: leader, follower, and tail, each moving as specified depending on its type. All relays are initially placed close to the first AP. Then, the relay leader moves forward until it finds the second AP. Each time the RSSI value falls below a given threshold, a follower relay will move to maintain the connectivity between the first AP and the leader; this follower will pursue the node ahead of it, creating a chain. Once the leader reaches the second AP, it will stop when it finds the best RSSI value, and the remaining relays will stop iteratively based on the same rule.

In the two aforementioned algorithms [16], [17], a differentiation among the mobile robots is assumed; i.e., there are leader robots and follower robots. Such a differentiation may be regarded as a weakness because the followers' motion depends on the leader's motion; thus, if the leader fails for any reason, it will lead to a failure in the remainder of the chain. The mobile autonomous router system (MARS) similarly enables mobile relays to self-deploy in a string-type formation; however, the main difference is that in MARS, all nodes are equal [18]. Each mobile relay adjusts its position depending on the link quality characterization in an attempt to achieve the best possible reception in terms of bandwidth.

Conversely, schemes of the second type are based on strategies that strive to spread the nodes to cover a given area as thoroughly as possible (see Figure 4). For instance, in [19], Timotheou and Loukas reported a robotic backbone deployment algorithm based on the locations of trapped civilians. The objective is to maximize the number of connected civilians to facilitate the establishment of communication between them and the rescue command center. The robots search for civilians and cooperate to maintain backbone connectivity. To this end, each robot controls its own movements by executing three basic steps: exploration, connectivity, and settling. During the first step, the robot explores the zone searching for civilians; the robot will stop moving if its movement breaks the connectivity of the network or if the robot achieves direct connectivity with civilians. In the latter case, the robot will become a cluster-head and assign the task of exploration to other robots. One disadvantage of this technique is that the authors assume that the robots have a priori knowledge of the disaster zone; however, such information is not always available in advance.

The work presented by Reich et al. [20] is of the same type. These authors consider a mobile network that automatically maintains its own connectivity by constantly moving its nodes. The authors propose an algorithm for the self-spreading of the mobile nodes over a given area. Each node, moving independently, uses two-hop radius knowledge to determine when to terminate its motion based on the decision criterion. This criterion represents the risk of dividing or disconnecting the network. Thus, each node executes the algorithm, which the authors name SCAN, in such a way as to maintain connectivity. The algorithm works as follows: if a given node still possesses at least a predefined number of link connections with its neighbors, then it continues moving; i.e., it increases its distance from its neighbors. Otherwise, the node must halt movement because any movement will incur a high probability of disconnection.

The mobile robotic approach considerably reduces the necessity for human intervention in network deployment. Moreover, node mobility allows for flexible deployment or even redeployment to adapt the network topology to different indoor and outdoor scenarios. In particular, nodes in the second type of scheme may be configured to spread while maintaining multi-path communication.

Finally, the SWaP requirements for this approach may increase considerably as a function of the size of the deployed network, especially with respect to power consumption because the nodes are mobile. Although the size and weight of each node might be very low, power consumption is a key performance factor. Thus, a manager implementing an impromptu network must have the ability to decide the type of network to be deployed almost in real time because such decisions are vital to the success of deployment.

\section{Discussion}

In this article, we presented an overview of rapid deployment solutions for the creation of post-disaster networks. This is a crucial task because communications between the rescue teams and the victims depend on this infrastructure replacement. We proposed a classification of the proposed approaches with the purpose of providing the interested reader with an outline of the topic. 
Hurricane Odile, which we cited at the beginning of this article, is just one example of the natural or man-made disasters for which the various solutions we surveyed for rapid network deployment may be well suited. The main differences among such solutions lie in the available resources and the abilities of the team that will deploy the impromptu network. For example, the difficulty of accounting for the infrastructure, coordination, and resources needed to deploy a metropolitan area network is considerably greater than that for the deployment of a local area network. The devices required to deploy a breadcrumbor mobile-robot-based network may be relatively easy for emergency services to obtain, whereas those necessary to deploy a metropolitan network should, in the ideal case, be compatible with the existing pre-established infrastructure.

In recent years, because of the ubiquity of mobile devices such as smartphones, laptops, and tablets, new possibilities regarding the usage of such devices in disaster scenarios have arisen. Such devices are usually provided with $\mathrm{Wi}-\mathrm{Fi}$, Bluetooth, or $3 \mathrm{G}$ or $4 \mathrm{G}$ radio interfaces and equipped with GPS receivers for self-positioning. All these characteristics are beneficial for enabling device-to-device communications, and the embedded sensors of such devices, such as video and still-image cameras, may potentially provide emergency teams with an accurate view of what is happening in the disaster zone. For example, the authors of [21] studied the feasibility of using smartphones as gateways/routers in wireless mesh networks. In [22], the proposed STEM-Net architecture is based on a WMN composed of survivors' personal wireless devices capable of self-adapting their configurations according to the needs of the network and working cooperatively with other devices to replace the portion of the infrastructure damaged by a disaster.

We believe that the development of a truly effective post-disaster network still requires further effort, and future proposed solutions must allow for flexible deployment or redeployment to adapt to the conditions of each scenario. In addition, such solutions should function with minimal human intervention over the incident area and should be compatible with current technologies to cooperate with the already-existing infrastructure without neglecting the issue of energy conservation, which is extremely critical in the case of battery-powered wireless devices.

\section{ACKNOWLEDGEMENT}

This work was partially supported by a grant from CPER Nord-Pas-de-Calais/FEDER Campus Intelligence Ambiante, the French National Research Agency (ANR) under the VERSO RESCUE project (ANR10-VERS-003), and from the Italian government within the Cooperlink initiative under the international research project "PALMARES: an Internet of Smart Objects".

\section{BIOGRAPHIES}

Karen Miranda (kmiranda@correo.cua.uam.mx) received the PhD degree in Computer Science from the University of Lille 1, France in 2013 and the MSc degree in Information Sciences and Technologies from the Metropolitan Autonomous University (UAM), Mexico in 2009. From January 2011 to April 2014, she worked within the FUN research team at Inria Lille - Nord Europe, France. Currently, she is a visiting scholar with the Department of Applied Mathematics and Systems at the Metropolitan Autonomous University (UAM) in Mexico City. Her major research interests fall into performance evaluation of computer protocols, robot-based wireless relay networks, and wireless sensor networks.

Antonella Molinaro has been an Associate Professor of Telecommunications with the University Mediterranea of Reggio Calabria, Italy, since 2005. Before, she was an Assistant Professor with the University of Messina (1998-2001), with the University of Calabria (2001-2004), and a research fellow at the Polytechnic of Milan (1997-1998). She was with Telesoft, Rome (1992-1993), and with Siemens, Munich (19941995) as a CEC Fellow in the RACE-II program. Her current research focuses onto wireless and mobile networking, vehicular networks, and future Internet.

Tahiry Razafindralambo received his MSc in applied statistics and computer science from the university of Antananarivo in 2001 and his PhD. degree in Computer Science from the INSA de Lyon in 2007. He is currently an Inria full researcher. His research interests are mainly focused on distributed algorithms and 
protocols design for wireless networks and performance evaluation. He is involved in many organization and program committees of national and international conferences such as DCOSS, MASS, PE-WASUN, MSWIM, PIMRC, ICC and he is the principal investigator of many national and international projects.

\section{REFERENCES}

[1] Y. Ran, "Considerations and suggestions on improvement of communication network disaster countermeasures after the Wenchuan earthquake," IEEE Communications Magazine, vol. 49, no. 1, pp. 44-47, Jan. 2011.

[2] K. Mase, "How to deliver your message from/to a disaster area," IEEE Communications Magazine, vol. 49, no. 1, pp. 52-57, Jan. 2011.

[3] J. Evans, G. Minden, K. S. Shanmugan et al., "The Rapidly Deployable Radio Network," IEEE Journal on Selected Areas in Communications, vol. 17, no. 4, pp. 689-703, Apr. 1999.

[4] F. Legendre, T. Hossmann, F. Sutton, and B. Plattner, "30 Years of Wireless Ad Hoc Networking Research: What about Humanitarian and Disaster Relief Solutions? What are we still missing?" in Proceedings of the 1st International Conference on Wireless Technologies for Humanitarian Relief (ACWR), Amritapuri, Kollam, Kerala, India, Dec. 2011, pp. 217-217.

[5] R. Bruno, M. Conti, and E. Gregori, "Mesh Networks: Commodity Multihop Ad Hoc Networks," IEEE Communications Magazine, vol. 43, no. 3, pp. 123-131, Mar. 2005.

[6] S. Ghafoor, P. Sutton, C. Sreenan, and K. Brown, "Cognitive radio for disaster response networks: survey, potential, and challenges," IEEE Wireless Communications, vol. 21, no. 5, pp. 70-80, Oct. 2014.

[7] M. Berioli, A. Molinaro, S. Morosi, and S. Scalise, "Aerospace communications for emergency applications," Proceedings of the IEEE, vol. 99, no. 11, pp. 1922-1938, Nov. 2011.

[8] H. Aïache, R. Knopp, K. Koufos, H. Salovuori, and P. Simon, "Increasing Public Safety Communications Interoperability: The CHORIST Broadband and Wideband Rapidly Deployable Systems," in Proceedings of the Next Generation Public Safety Communication Networks and Technologies (NGenSafe), Dresden, Germany, Jun. 2009.

[9] H. Aïache, V. Conan, G. Guibé et al., "WIDENS: Advanced Wireless Ad-Hoc Networks for Public Safety," in Proceedings of the IST Mobile \& Wireless Communications Summit, Dresden, Germany, Jun. 2005.

[10] E. Cayirci and C. Ersoy, "Application of 3G PCS technologies to rapidly deployable mobile networks," IEEE Networks, vol. 16, pp. 20-27, 2002

[11] E. Del Re, S. Jayousi, S. Morosi, L. S. Ronga, M. De Sanctis, E. Cianca, M. Ruggieri, E. Falletti, A. Iera, G. Araniti, and C. Sacchi, "SALICE project: Satellite-Assisted Localization and Communication Systems for Emergency Services," IEEE Aerospace and Electronic Systems Magazine, vol. 28, no. 9, pp. 4-15, Sep. 2013.

[12] M. R. Souryal, J. Geissbuehler, L. E. Miller, and N. Moayeri, "Real-Time Deployment of Multihop Relays for Range Extension," in Proceedings of the 5th International Conference on Mobile Systems, Applications, and Services (MobiSys), San Juan, Puerto Rico, Jun. 2007, pp. 85-98.

[13] A. Wolff, S. Subik, and C. Wietfeld, "Performance Analysis of Highly Available Ad Hoc Surveillance Networks Based on Dropped Units," in Proceedings of IEEE Conference on Technologies for Homeland Security, Waltham, MA, USA, May 2008, pp. $123-128$.

[14] J. Q. Bao and W. C. Lee, "Rapid deployment of wireless ad hoc backbone networks for public safety incident management," in Proceedings of the Global Communications Conference (GLOBECOM), Nov. 2007, pp. 1217-1221.

[15] H. Liu, Z. Xie, J. Li, S. Lin, D. Siu, P. Hui, K. Whitehouse, and J. A. Stankovic, "An Automatic, Robust, and Efficient Multi-User Breadcrumb System for Emergency Response Applications," IEEE Transactions on Mobile Computing, vol. PP, no. $99,2013$.

[16] N. Pezeshkian, H. G. Nguyen, and A. Burmeister, "Unmanned ground vehicle radio relay deployment system for non-line-of-sight operations," in Proceedings of the 13th IASTED International Conference on Robotics and Applications, Würzburg, Germany, 2007, pp. 501-506.

[17] C. Q. Nguyen, B.-C. Min, E. T. Matson, A. H. Smith, J. E. Dietz, and D. Kim, "Using Mobile Robots to Establish Mobile Wireless Mesh Networks and Increase Network Throughput," International Journal of Distributed Sensor Networks (IJDSN), vol. 2012, pp. $1-13,2012$.

[18] K.-H. Kim, K. G. Shin, and D. Niculescu, "Mobile Autonomous Router System for Dynamic (Re)formation of Wireless Relay Networks," IEEE Transactions on Mobile Computing, vol. 12, no. 9, pp. 1828-1841, 2013.

[19] S. Timotheou and G. Loukas, "Autonomous networked robots for the establishment of wireless communication in uncertain emergency response scenarios," in Proceedings of the 2009 ACM Symposium on Applied Computing (SAC), Honolulu, Hawaii, USA, Mar. 2009, pp. $1171-1175$.

[20] J. Reich, V. Misra, D. Rubenstein, and G. Zussman, "Connectivity Maintenance in Mobile Wireless Networks via Constrained Mobility," IEEE Journal on Selected Areas in Communications, vol. 30, no. 5, pp. 935-950, Jun. 2012.

[21] A. Iera, A. Molinaro, S. Y. Paratore, G. Ruggeri, and A. Zurzolo, "Making a mesh router/gateway from a smartphone: Is that a practical solution?" Ad Hoc Networks, vol. 9, no. 8, pp. 1414-1429, Nov. 2011.

[22] G. Aloi, L. Bedogni, M. Di Felice, V. Loscrì, A. Molinaro, E. Natalizio, P. Pace, G. Ruggeri, A. Trotta, and N. R. Zema, "STEM-Net: an evolutionary network architecture for smart and sustainable cities," Transactions on Emerging Telecommunications Technologies, vol. 25, no. 1, pp. 21-40, Jan. 2014. 


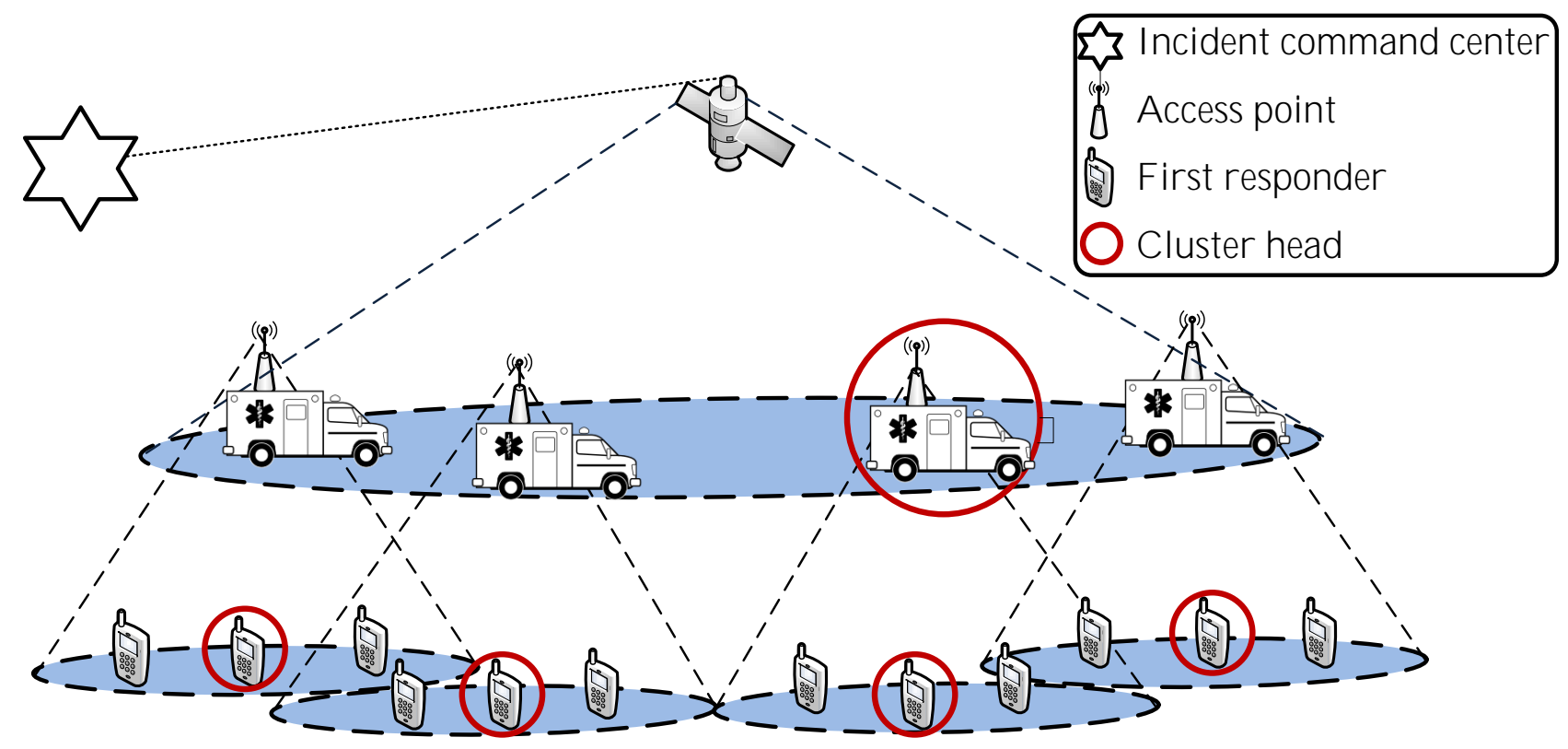

Fig. 1: A metropolitan area approach deployment scheme based on clustering networks. 


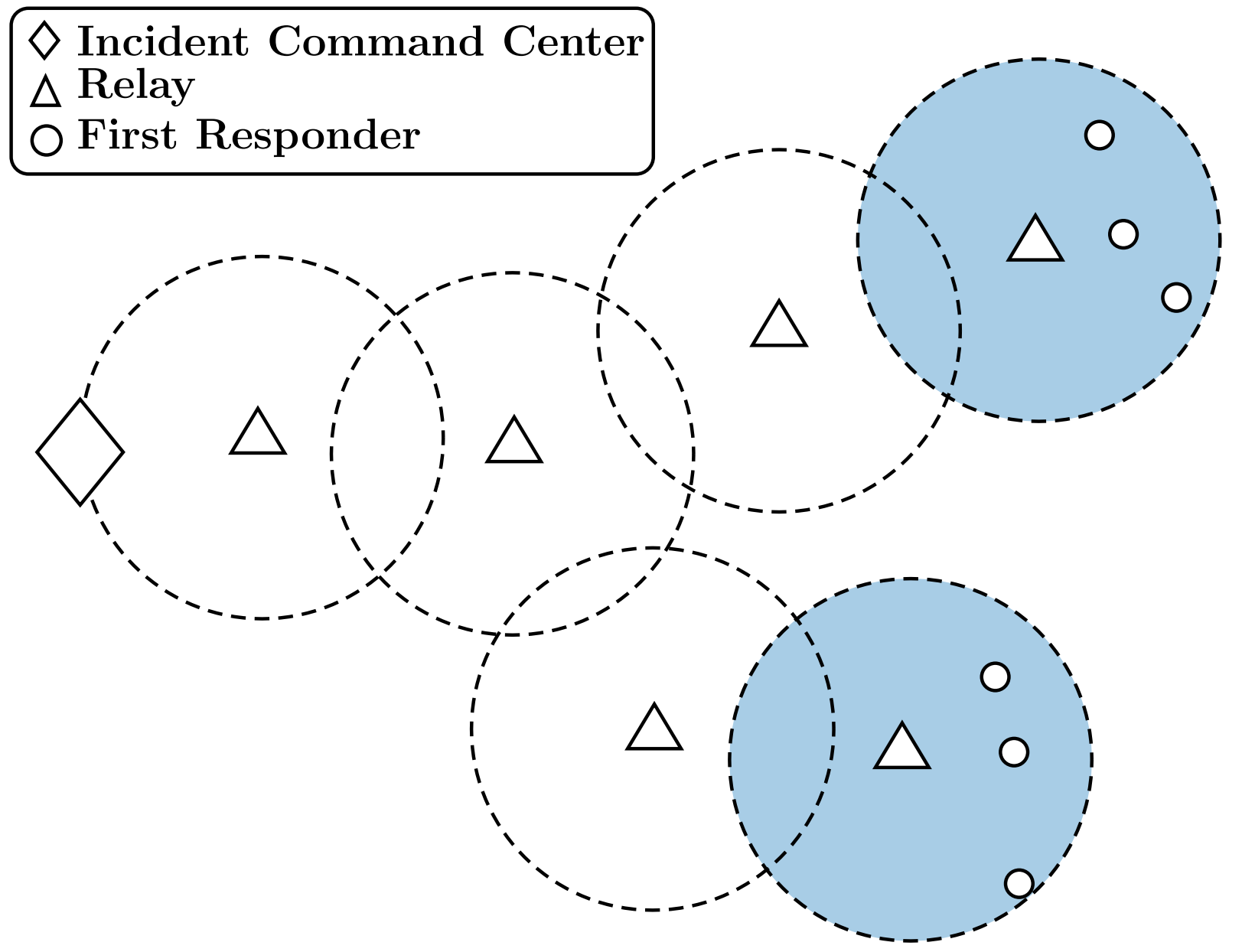

Fig. 2: A conceptual schema of a breadcrumb-based network. 


\section{Initial configuration}
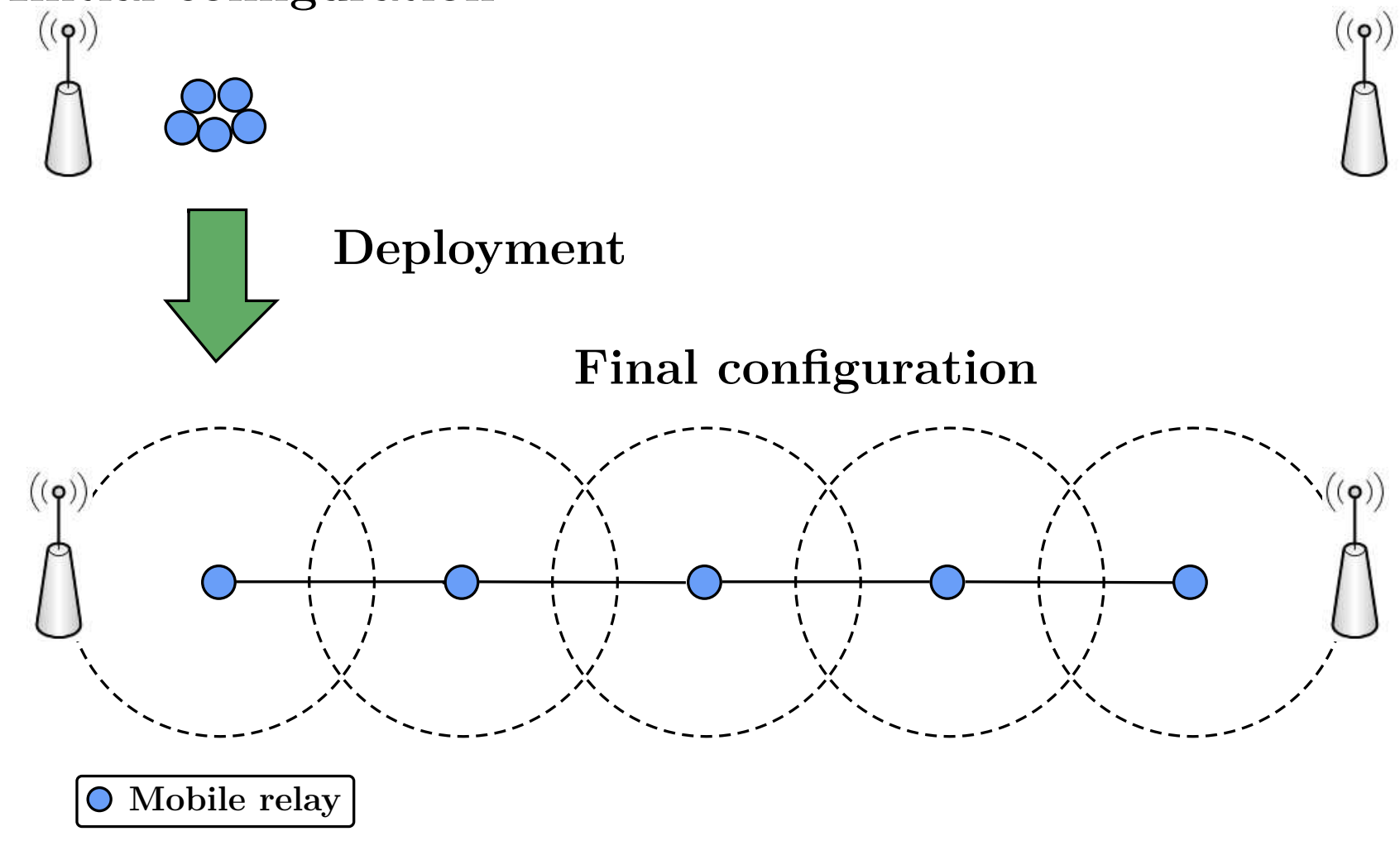

Fig. 3: Chain-like deployment of a mobile robotic backbone-based network. 


\section{Initial configuration}
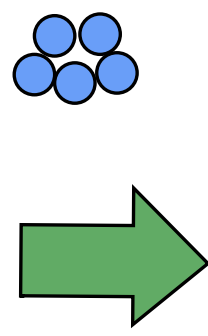

Deployment

O Mobile relay

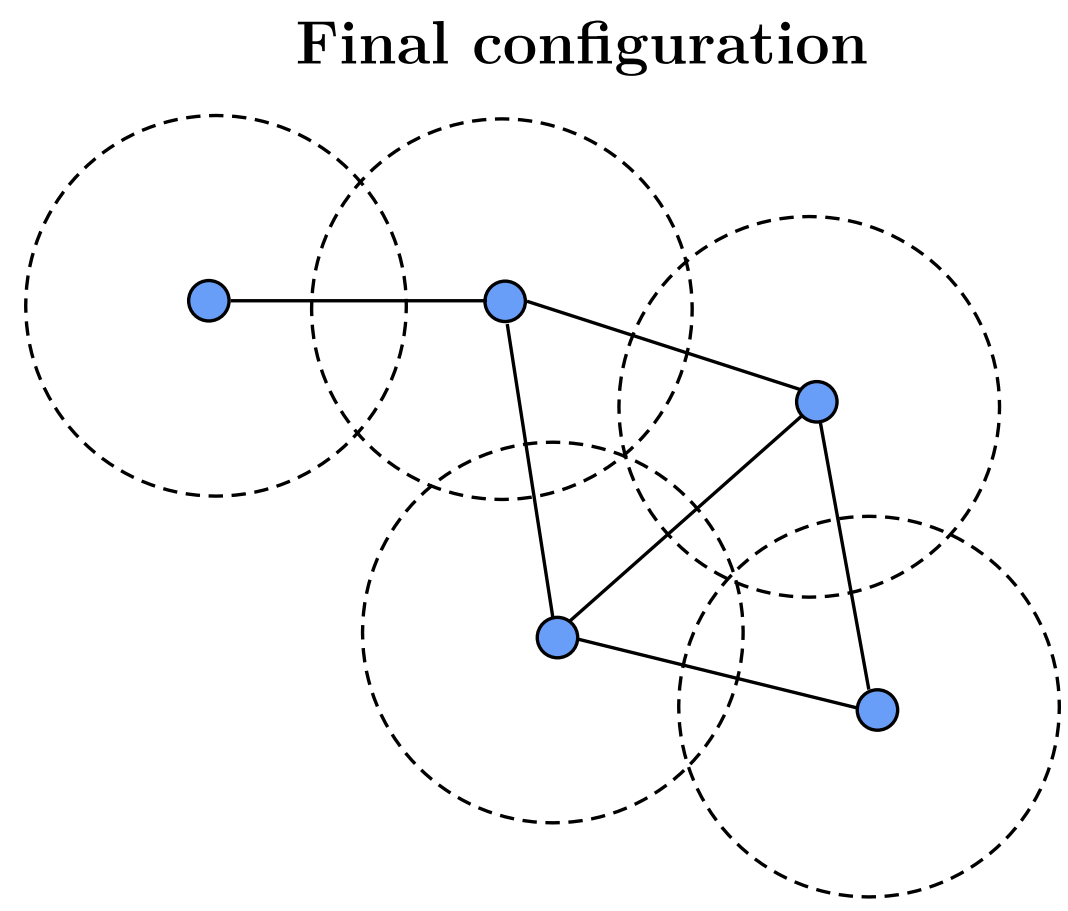

Fig. 4: Spread deployment of a mobile robotic backbone-based network. 